\title{
(6) OPEN ACCESS \\ Duration and breaks in sedentary behaviour: accelerometer data from 1566 community-dwelling older men (British Regional Heart Study)
}

\author{
Barbara J Jefferis, ${ }^{1,2}$ Claudio Sartini, ${ }^{1}$ Eric Shiroma, ${ }^{3}$ Peter $\mathrm{H}$ Whincup, ${ }^{4}$ \\ S Goya Wannamethee, ${ }^{1}$ I-Min Lee ${ }^{3}$
}

\begin{abstract}
${ }^{1}$ Department of Primary Care \& Population Health, University College London Medical School, London, UK ${ }^{2}$ Physical Activity Research Group, University College London, London, UK ${ }^{3}$ Brigham and Women's Hospital, Harvard Medical School, Boston, Massachusetts, USA

${ }^{4}$ Division of Population Health Sciences and Education, St George's University of London, London, UK
\end{abstract}

Correspondence to Dr Barbara I Jefferis, Department of Primary Care \& Population Health, University College London Medical School, Rowland Hill Street, London NW3 2PF, UK; b.jefferis@ucl.ac.uk

Accepted 3 September 2014 Published Online First 17 September 2014

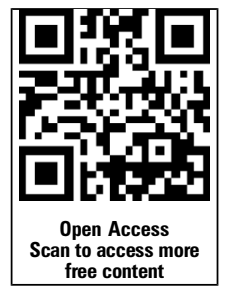

\section{ABSTRACT}

Background Sedentary behaviours are increasingly recognised as raising the risk of cardiovascular disease events, diabetes and mortality, independently of physical activity levels. However, little is known about patterns of sedentary behaviour in older adults.

Methods Cross-sectional study of 1566/3137 (50\% response) men aged 71-91 years from a UK populationbased cohort study. Men wore a GT3x accelerometer over the hip for 1 week in 2010-2011. Mean daily minutes of sedentary behaviours, percentage of day in sedentary behaviours, sedentary bouts and breaks were calculated and summarised by health and demographic characteristics.

Results 1403 ambulatory men aged 78.4 years ( $S D=4.6$ years) with $\geq 600$ min of accelerometer wear on $\geq 3$ days had complete data on covariables. Men spent on average $618 \mathrm{~min}(\mathrm{SD}=83)$, or $72 \%$ of their day in sedentary behaviours ( $<100$ counts $/ \mathrm{min})$. On average, men accumulated 72 spells of sedentary behaviours per day, with 7 breaks in each sedentary hour. Men had on average 5.1 sedentary bouts of $\geq 30 \mathrm{~min}$, which accounted for $43 \%$ of sedentary time, and 1.4 bouts of $\geq 60 \mathrm{~min}$, which accounted for $19 \%$ of daily sedentary time. Men who were over 80 years old, obese, depressed and had multiple chronic conditions accumulated more sedentary time and spent more time in longer sedentary bouts.

Conclusions Older men spend nearly three quarters of their day in sedentary behaviours, mostly accumulated in short bouts, although bouts lasting $\geq 30$ min accounted for nearly half of the sedentary time each day. Men with medical risk factors were more likely to also display sedentary behaviour.

\section{INTRODUCTION}

Sedentary behaviours are increasingly recognised as raising the risk of cardiovascular disease (CVD) events, diabetes and mortality, independently of physical activity (PA) levels, ${ }^{1}{ }^{2}$ although the independence has been questioned. ${ }^{3}$ Sedentary behaviours can be defined as activities with an energy expenditure under 1.5 METS (metabolic equivalents) and a sitting or reclining posture while awake. ${ }^{4}$ New UK guidelines ${ }^{5}$ suggest that 'long periods' of sedentary behaviours should be avoided, but they do not provide clear guidance about how long such periods are, nor how often to break them up. Most research has focused on the total time spent in sedentary behaviours each day, or proxies, including the number of hours per day spent watching television, yet television or screen time captures only a fraction of the daily sedentary behaviours.

The recent availability of instruments to measure PA and sedentary behaviours objectively permits a more detailed investigation of patterns of sedentary behaviours, including the duration of spells and frequency of breaks in sedentary time. Older adults (over 65 years) are the most sedentary age group ${ }^{6}$ and also the most rapidly growing age group in the UK and other developed countries. However, little is known about the actual patterns of sedentary behaviour in older adults; such data could be important for targeting interventions if breaking up sedentary behaviour is demonstrated to reduce clinical disease and mortality, and bouts of a particular duration are associated with negative outcomes. A recent study ${ }^{7}$ reported on accelerometer measured sedentary behaviour patterns and associations with key lifestyle factors in older women. There are few comparable data available in older men. ${ }^{8}$ We therefore investigated patterns of sedentary behaviour in relation to these variables, as well as physical and mental health status, which are known to be predictors of low PA levels in older men. ${ }^{9}$

\section{METHODS}

The British Regional Heart Study is a prospective cohort study of 7735 men recruited from a single Primary care centre in 24 British towns in 19781980 (age 40-59 years). Since 2010, survivors have been invited to participate in annual studies of objectively measured PA. We use cross-sectional data collected between 2010 and 2012, when 3137 survivors were invited to a clinic visit and asked to participate in a study of objectively measured PA. Local research ethics committees provided ethical approval and participants gave written informed consent. Participants wore a GT3X accelerometer (Actigraph, Pensacola, Florida, USA) over the hip for 7 days, during waking hours, removing it while bathing. Data collected were processed using standard methods; raw data collected from movements registering on the vertical axis were integrated into $60 \mathrm{~s}$ increments, called epochs. Non-wear time was identified and excluded using a commonly used and freely available R package 'Physical Activity'. ${ }^{10}$ Periods of continuous zeros lasting more than $90 \mathrm{~min}$ were assigned as non-wear time; short spells of non-zero counts lasting up to $2 \mathrm{~min}$ during the $90 \mathrm{~min}$ period were allowed as non-wear time if no activity counts were detected during the $30 \mathrm{~min}$ before and after that interval, to reflect the possibility of the monitor accidentally 
being moved when not being worn. This means that any non-zero counts except the allowed short interval of up to 2 min are considered as wear time.

The number of minutes per day spent in sedentary behaviour was categorised using standard count-based intensity threshold values of counts per minute: $<100$ for sedentary behaviour $\left(<1.5\right.$ MET). ${ }^{11}$ Three further summary measures of sedentary behaviours were calculated per day and averaged over valid days (1) percentage of the wear-day spent in sedentary behaviour (2) number of sedentary bouts (defined as a period of consecutive minutes where the accelerometer registers $<100$ counts/min) (3) number of sedentary breaks (defined as at least $1 \mathrm{~min}$ where the accelerometer registers $\geq 100$ counts/min following a sedentary bout).

Nurses measured height and weight at a clinic visit. Men completed questionnaires including data on cigarette smoking (current, ex, never), presence of chronic diseases $(0,1-2$ or $\geq 3$ of the following: heart attack, heart failure, angina, other heart trouble, diabetes, stroke, osteoporosis, arthritis and claudication) and depression (scoring $\geq 2$ on the 4 -item Geriatric Depression Scale). ${ }^{12}$

Summary measures of sedentary behaviours were calculated according to age, body mass index (BMI), smoking status, chronic diseases and depression, using adjusted marginal means from mixed models (stata xtmixed) adjusted for age, BMI, smoking status, wear time (continuous variables) and day of week (weekdays, Saturday, Sunday), smoking status, depression, and chronic diseases (categorical variables). Differences across each measure of sedentary behaviour compared to the baseline group were tested. Complete case analysis was used, that is, with data available for the outcome (three or more days of data) and all covariates. Statistical analyses were conducted in R and Stata V.13. ${ }^{13} 14$

\section{RESULTS}

Of $3137,1566(50 \%)$ survivors who were invited agreed to participate and provided accelerometer data. Of these, 1455/1566 (93\%) were independently mobile community-dwelling men, had completed the questionnaire and, by convention, provided $\geq 600$ min wear time on $\geq 3$ days. ${ }^{11} 1403 / 1455$ men with a mean age of 78.4 years $(S D=4.6)$ had data on BMI, smoking, depression and presence of chronic diseases. Men had a mean of $6.7(\mathrm{SD}=0.8)$ valid wear days and spent a mean of $618 \mathrm{~min}$ $(\mathrm{SD}=83), 72 \%$ of their wear-day, in sedentary behaviours. The percentage wear time spent in sedentary behaviours was higher at older age, particularly in the over 80s, at higher BMI, especially the obese, in men who were depressed and in men with a higher number of chronic health conditions (table 1).

There were on average 72 sedentary bouts per day (lasting median 3.2 min IQR 1.1-9.9), with on average 7 breaks per hour of sedentary behaviour. The number of bouts and breaks per hour decreased (indicating more time spent in longer sedentary bouts) in men who were older, had a higher BMI, more chronic conditions and depression. Smokers had more sedentary bouts per day than non-smokers. On average, men had 5.1 bouts per day of sedentary behaviour lasting $\geq 30$ min, which accounted for $43 \%$ of sedentary time, and 1.4 only sedentary bouts per day lasting $\geq 60 \mathrm{~min}$, yet these accounted for $19 \%$ of daily sedentary time (table 2 ).

Table 1 Characteristics of sedentary behaviour in 1403 men in the British Regional Heart Study

\begin{tabular}{|c|c|c|c|c|c|c|c|c|c|}
\hline & \multirow{2}{*}{$\begin{array}{l}\text { Number of } \\
\text { men }(\%)\end{array}$} & \multicolumn{2}{|l|}{$\begin{array}{l}\text { Sedentary behaviour } \\
\text { (min/day) }\end{array}$} & \multicolumn{2}{|c|}{$\begin{array}{l}\text { Wear time in sedentary } \\
\text { behaviour* }(\%)\end{array}$} & \multicolumn{2}{|c|}{$\begin{array}{l}\text { Number of bouts sedentary } \\
\text { behaviour/dayt }\end{array}$} & \multicolumn{2}{|c|}{$\begin{array}{l}\text { Number of breaks per } \\
\text { sedentary hour }\end{array}$} \\
\hline & & Mean $(95 \% \mathrm{Cl}) \S$ & p Valueף & Mean $(95 \% \mathrm{Cl}) \S$ & p Valueף & Mean $(95 \% \mathrm{Cl}) \S$ & p Valueף & Mean $(95 \% \mathrm{Cl}) \S$ & p Valuen \\
\hline All men $\S^{\prime * *}$ & 1403 & 618 (614 to 621$)$ & & 72.4 (72.0 to 72.8$)$ & & 71.9 (71.2 to 72.6$)$ & & 7.2 (7.1 to 7.3$)$ & \\
\hline Age group & & & $<0.001$ & & $<0.001$ & & $<0.001$ & & $<0.001$ \\
\hline 70 to $<75$ years & $389(27.7)$ & 602 (595 to 609) & & 69.7 (68.9 to 70.5$)$ & & 73.4 (72.2 to 74.6$)$ & & 7.6 (7.4 to 7.8$)$ & \\
\hline 75 to $<80$ years & $544(38.8)$ & 610 (603 to 616$)$ & & 71.2 (70.5 to 72.0$)+t$ & & 72.7 (71.6 to 73.8 ) & & 7.4 (7.2 to 7.6$)$ & \\
\hline$\geq 80$ years & $470(33.5)$ & 641 (635 to 647)†† & & 76.0 (75.3 to 76.7$)+\dagger$ & & $69.6(68.3$ to 70.9$)+\dagger$ & & $6.7(6.5$ to 6.9$)+\dagger$ & \\
\hline BMI $\left(\mathrm{kg} / \mathrm{m}^{2}\right)$ & & & 0.002 & & $<0.001$ & & $<0.001$ & & $<0.001$ \\
\hline$<25$ & $406(28.9)$ & 614 (606 to 621$)$ & & 71.5 (70.6 to 72.3 ) & & 74.5 (73.2 to 75.8$)$ & & 7.5 (7.3 to 7.7$)$ & \\
\hline 25 to $<30$ & $722(51.5)$ & 613 (608 to 618 ) & & 71.7 (71.1 to 72.3 ) & & 72.7 (71.7 to 73.7 ) & & 7.4 (7.2 to 7.5$)$ & \\
\hline$\geq 30$ & $275(19.6)$ & 637 (629 to 644)t† & & 75.7 (74.8 to 76.5$)+\dagger$ & & $65.7(64.1$ to 67.3$)+\dagger$ & & $6.4(6.2$ to 6.6$)+\dagger$ & \\
\hline Smoking status & & & 0.012 & & 0.073 & & 0.008 & & 0.297 \\
\hline Non-smoker & $1356(96.7)$ & 617 (613 to 620$)$ & & 72.3 (71.9 to 72.7 ) & & 71.7 (71.0 to 72.4 ) & & 7.2 (7.1 to 7.3$)$ & \\
\hline Smoker & $47(3.3)$ & 649 (627 to 671)†† & & 74.9 (72.4 to 77.4$)$ & & $77.6(73.3$ to 82.0$)+\dagger$ & & 7.5 (6.9 to 8.2$)$ & \\
\hline Depression & & & $<0.001$ & & $<0.001$ & & $<0.001$ & & $<0.001$ \\
\hline Not depressed & $1094(78.0)$ & 613 (609 to 617 ) & & 71.3 (70.8 to 71.8 ) & & 73.1 (72.3 to 73.9 ) & & 7.4 (7.3 to 7.5$)$ & \\
\hline Depressed & $309(22.0)$ & 634 (626 to 642)t† & & 76.4 (75.4 to 77.3$)+\dagger$ & & $67.4(65.8$ to 68.9$)+\dagger$ & & $6.6(6.4$ to 6.8$)+\dagger$ & \\
\hline Chronic conditions & & & $<0.001$ & & $<0.001$ & & $<0.001$ & & $<0.001$ \\
\hline None & $657(46.8)$ & 621 (615 to 627$)$ & & 73.0 (72.3 to 73.7 ) & & 71.3 (70.1 to 72.5$)$ & & 7.1 (6.9 to 7.3 ) & \\
\hline $1-2$ & $644(45.9)$ & $630(619$ to 640$)+\dagger$ & & 74.4 (73.1 to 75.6$) \mathrm{t \dagger}$ & & $69.4(67.4$ to 71.4$)+\dagger$ & & $6.9(6.6$ to 7.2$)+\dagger$ & \\
\hline$\geq 3$ & $102(7.3)$ & 652 (637 to 667$)+\dagger$ & & 78.3 (76.5 to 80.1$)+\dagger$ & & $66.6(63.3$ to 70.0$) \mathrm{t \dagger}$ & & $6.3(5.9$ to 6.8$)+\dagger$ & \\
\hline
\end{tabular}

\footnotetext{
*Percentage sedentary=100xsedentary (<100 cpm) minutes/total wear time (min).

$\dagger \mathrm{A}$ bout of sedentary behaviour is a period of consecutive minutes where the accelerometer registers $<100 \mathrm{cpm}$

¥A break in sedentary behaviour is at least one minute where the accelerometer registers $>100 \mathrm{cpm}$ following a sedentary bout.

$\S$ Marginal means are adjusted for age, BMI, smoking status, wear time, day order of week, number of chronic diseases and depression.

Ip Value tests for equal means across all levels of the subgroup.

${ }_{* \star}$ Ambulatory men, not in a care home, no missing covariate data.

t†Pairwise mean difference $\mathrm{p}<0.05$ when compared to age $<75$, BMI $<25$, non-smoker, not depressed, or 0 chronic conditions.

BMI, body mass index.
} 
Table 2 Number of sedentary bouts/day, percentage of sedentary bouts and percentage of sedentary time of various bout durations $(n=1403)$

\begin{tabular}{llcc}
\hline $\begin{array}{l}\text { Bout } \\
\text { duration } \\
\text { (min) }\end{array}$ & $\begin{array}{l}\text { Number of } \\
\text { sedentary bouts per } \\
\text { day, mean (SD)* }\end{array}$ & $\begin{array}{l}\text { Sedentary } \\
\text { bouts, mean } \\
\text { (SD) (\%) } \dagger\end{array}$ & $\begin{array}{l}\text { Sedentary time, } \\
\text { mean (SD) (\%)‡ }\end{array}$ \\
\hline $1+$ & $71.6(15.7)$ & 100 & 100 \\
$5+$ & $27.5(4.6)$ & $39.6(7.6)$ & $86.5(5.0)$ \\
$10+$ & $16.5(2.7)$ & $24.3(7.2)$ & $74.5(8.4)$ \\
$20+$ & $8.5(1.9)$ & $12.9(5.8)$ & $56.5(11.8)$ \\
$30+$ & $5.1(1.6)$ & $8(4.7)$ & $43.2(13.2)$ \\
$40+$ & $3.3(1.3)$ & $5.2(3.8)$ & $33.1(13.2)$ \\
$50+$ & $2.1(1.1)$ & $3.5(3.1)$ & $25.1(12.7)$ \\
$60+$ & $1.4(0.9)$ & $2.4(2.6)$ & $18.8(11.7)$ \\
$70+$ & $0.9(0.7)$ & $1.6(2.2)$ & $14.1(10.7)$ \\
$80+$ & $0.6(0.6)$ & $1.1(1.9)$ & $10.5(9.6)$ \\
$90+$ & $0.4(0.4)$ & $0.8(1.7)$ & $7.7(8.5)$ \\
$100+$ & $0.3(0.4)$ & $0.6(1.5)$ & $5.8(7.5)$ \\
$110_{+}$ & $0.2(0.3)$ & $0.4(1.4)$ & $4.4(6.6)$ \\
$120+$ & $0.1(0.2)$ & $0.3(1.3)$ & $3.3(6.1)$ \\
\hline
\end{tabular}

${ }^{*} \mathrm{~A}$ bout of sedentary behaviour is a period of consecutive minutes where the accelerometer registers $<100 \mathrm{cpm}$.

tPercentage of sedentary bouts, number of sedentary bouts of $n$ min/number of sedentary bouts of $1 \mathrm{~min}$.

¥Percentage of sedentary time, length of sedentary bouts of $n$ min/length of sedentary bouts of $1 \mathrm{~min}$.

\section{DISCUSSION}

These are the first detailed data on patterns of sedentary behaviours (number and duration of bouts, breaks and total amount of sedentary behaviours) in older men, complementing published data on the same measures of sedentary behaviours in older women in the USA ${ }^{7}$ and data on the total amount of sedentary behaviours in men and women in Iceland. ${ }^{8}$

In this large community-based sample of older men, nearly three quarters of the day when the monitor was being worn was spent in sedentary behaviours. On average, sedentary behaviour was broken up frequently with around seven breaks per sedentary hour. However, a very small proportion $(2.4 \%)$ of the total number of sedentary bouts per day lasted $1 \mathrm{~h}$ or more, and yet they filled large portions of the day, accounting for one fifth of total sedentary time.

Older men who were depressed, smokers, obese or suffered more chronic health conditions spent more of the day in prolonged sedentary spells; hence, they, who already have characteristics which increase risks of CVD and diabetes, may also bear a heavier health burden from their sedentary behaviours.

The percentage of the day spent in sedentary behaviours reported here was similar but very slightly higher than for men of a similar age, ${ }^{8}$ and somewhat higher than among women, both of younger and of similar age in studies using the same brand of accelerometer worn over the hip. ${ }^{7}$ In our sample of men, we found that the number of breaks was lower and the duration of bouts was longer than reported in women. ${ }^{7}$ Hence, although older men are reported to have higher levels of moderate-to-vigorous physical activity than women, ${ }^{8} 9$ they may also have a higher total sedentary time and longer periods of sedentary behaviour.

The study benefits from including a large sample of community-dwelling, ambulatory older men rather than a highrisk clinical population (eg, nursing home residents), which should improve the generalisability of the results. However, it is possible that the study responders were more active and less sedentary than the non-responders. Indeed, compared with the men who declined to participate, men who participated were younger and reported being more active 10 years previously and had a lower BMI. Hence, the levels of sedentary behaviours reported here may be conservative estimates. Nevertheless, the $50 \%$ response rate to the postal accelerometer study compares favourably with prior population-based studies of older adults: $21 \%,{ }^{15} 43 \%{ }^{16}$ and in the over 75 -year-olds in the Health Survey for England, 37\% women and 48\% men had $\geq 4$ days with valid data. ${ }^{17}$ While our sample reflects patterns of sedentary behaviours in the UK, it may not reflect patterns in other countries. Finally, as we present cross-sectional data, we cannot infer causality.

While other data indicate that older adults do benefit from higher levels of activity, in terms of healthier ageing and also reduced levels of CVD and mortality, ${ }^{18-20}$ if breaking up sedentary behaviour is also demonstrated to reduce clinical disease and mortality, and bouts of particular duration are associated with negative outcomes, these data will help to target interventions. Our data suggest that the elderly suffering from multimorbidity, the obese, the depressed and smokers will require extra focus to reduce long sedentary bouts.

Acknowledgements Authors acknowledge the British Regional Heart Study team (Lucy Lennon and Sarah Ash) for data collection.

Contributors BJJ, CS, PHW and SGW acquired the data; I-ML, ES and BJJ conceived the study; CS and BJJ conducted the data analyses. BJJ, CS, ES, PHW, SGW and I-ML helped to interpret the data for the work and contributed to the drafting of the work and its critical revision for important intellectual content. All authors gave final approval of the version to be published.

Competing interests BJJ and CS were funded by an NIHR Post-Doctoral Fellowship (2010-03-023) to BJJ. The British Regional Heart study is supported by a British Heart Foundation programme grant (RG/08/013/25942). I-ML is supported in part by a US National Institutes of Health grant (CA154647).

Ethics approval National Research Ethics Service (NRES) Committee LondonCentral.

\section{Provenance and peer review Not commissioned; externally peer reviewed.}

Open Access This is an Open Access article distributed in accordance with the Creative Commons Attribution Non Commercial (CC BY-NC 3.0) license, which permits others to distribute, remix, adapt, build upon this work non-commercially, and license their derivative works on different terms, provided the original work is properly cited and the use is non-commercial. See: http://creativecommons.org/ licenses/by-nc/3.0/

\section{REFERENCES}

1 Gennuso KP, Gangnon RE, Matthews CE, et al. Sedentary behavior, physical activity, and markers of health in older adults. Med Sci Sports Exerc 2013;45:1493-500.

2 Ford ES, Caspersen CJ. Sedentary behaviour and cardiovascular disease: a review of prospective studies. Int J Epidemiol 2012;41:1338-53.

3 Maher C, Olds T, Mire E, et al. Reconsidering the sedentary behaviour paradigm. PLOS ONE 2014;9:e86403.

4 Sedentary Behaviour Research Network. Letter to the editor: standardized use of the terms sedentary and sedentary behaviours. Appl Physiol Nutr Metab 2012:37:540-2.

5 Chief Medical Officers of England, Scotland, Wales and Northern Ireland. Start active, stay active. A report on physical activity for health from the four home countries' Chief Medical Officers. London: Crown, 2011

6 Matthews CE, Chen KY, Freedson PS, et al. Amount of time spent in sedentary behaviors in the United States, 2003-2004. Am J Epidemiol 2008;167:875-81.

7 Shiroma EJ, Freedson PS, Trost SG, et al. Patterns of accelerometer-assessed sedentary behavior in older women. JAMA 2013;310:2562-3.

8 Arnardottir NY, Koster A, Van Domelen DR, et al. Objective measurements of daily physical activity patterns and sedentary behaviour in older adults: Age, Gene/ Environment Susceptibility-Reykjavik Study. Age Ageing 2013;42:222-9.

9 Jefferis BJ, Sartini C, Lee IM, et al. Adherence to physical activity guidelines in older adults using objectively measured physical activity in a population study. BMC Public Health 2014;14:382. 
10 Physical activity: process physical activity accelerometer data. Nashville, TN: Leena Choi, 2011.

11 Tudor-Locke C, Camhi SM, Troiano RP. A catalog of rules, variables, and definitions applied to accelerometer data in the National Health and Nutrition Examination Survey, 2003-2006. Prev Chronic Dis 2012;9:E113.

12 van Marwijk HW, Wallace P, de Bock GH, et al. Evaluation of the feasibility, reliability and diagnostic value of shortened versions of the geriatric depression scale. Br J Gen Pract 1995:45:195-9.

13 R: A Language and Environment for Statistical Computing Vienna, Austria: R Foundation for Statistical Computing, 2013.

14 Stata Statistical Software: Release 13 College Station, TX: StataCorp LP, 2013.

15 Davis MG, Fox KR, Hillsdon M, et al. Objectively measured physical activity in a diverse sample of older urban UK adults. Med Sci Sports Exerc 2011;43:647-54.
16 Harris $\mathrm{TJ}$, Owen CG, Victor CR, et al. What factors are associated with physical activity in older people, assessed objectively by accelerometry? Br I Sports Med 2009;43:442-50.

17 Craig R, Mindell J, Hirani V. Health Survey for England 2008. Physical activity and fitness. Summary of key findings. London: The Health and Social Care Information Centre, 2009.

18 Hamer M, Lavoie KL, Bacon SL. Taking up physical activity in later life and healthy ageing: the English longitudinal study of ageing. Br I Sports Med 2014;48:239-43.

19 Almeida OP, Khan KM, Hankey GJ, et al. 150 minutes of vigorous physical activity per week predicts survival and successful ageing: a population-based 11-year longitudinal study of 12201 older Australian men. Br J Sports Med 2014;48:220-5.

20 Ekblom-Bak E, Ekblom B, Vikstrom M, et al. The importance of non-exercise physical activity for cardiovascular health and longevity. Br J Sports Med 2014;48:233-8. 